\title{
Localisation fréquentielle des paquets d'ondelettes
}

Eric Séré

\begin{abstract}
Orthonormal bases of wavelet packets constitute a powerful tool in signal compression. It has been proved by Coifman, Meyer and Wickerhauser that "many" wavelet packets $w_{n}$ suffer a lack of frequency localization. Using the $L^{1}$-norm of the Fourier transform $\hat{w}_{n}$ as localization criterion, they showed that the average $2^{-j} \sum_{n=0}^{2^{j}-1}\left\|\hat{w}_{n}\right\|_{L^{1}}$ blows up as $j$ goes to infinity. A natural problem is then to know which values of $n$ create this blow-up in average. The present work gives an answer to this question, thanks to sharp estimates on $\left\|\hat{w}_{n}\right\|_{L^{1}}$ which depend on the dyadic expansion of $n$, for several types of filters. Let us point out that the value of $\left\|\hat{w}_{n}\right\|_{L^{1}}$ is a weak localization criterion, which can only lead to a lower estimate on the variance of $\hat{w}_{n}$.
\end{abstract}

\section{Résumé.}

Les bases orthonormées de paquets d'ondelettes sont un outil puissant en compression du signal. Coifman, Meyer et Wickerhauser ont prouvé que de "nombreux" paquets d'ondelettes $w_{n}$ souffrent d'une mauvaise localisation fréquentielle. Utilisant la norme $L^{1}$ de la transformée de Fourier $\hat{w}_{n}$ comme critère de localisation, ils ont montré que la moyenne $2^{-j} \sum_{n=0}^{2^{j}-1}\left\|\hat{w}_{n}\right\|_{L^{1}}$ explose lorsque $j$ tend vers l'infini. Il est alors naturel de se demander quelles valeurs de $n$ sont à l'origine de cette explosion en moyenne. Le présent travail donne une réponse à cette question, grâce à des estimations précises sur $\left\|\hat{w}_{n}\right\|_{L^{1}}$ en fonction du développement dyadique de $n$, et pour plusieurs types de filtres. 
Soulignons le fait que la valeur de $\left\|\hat{w}_{n}\right\|_{L^{1}}$ est un critère faible de localisation, qui ne peut conduire qu'à une estimation inférieure sur la variance de $\hat{w}_{n}$.

\section{Introduction.}

Les paquets d'ondelettes ont été introduits récemment en traitement et en compression du signal, où ils se sont révélés d'une grande efficacité (voir [2], [3]). On peut les considérer comme une réponse partielle au problème des bases d'atomes temps-fréquence (voir [7]).

Un atome temps-fréquence est une fonction du temps $f_{R}(t)$ associée à un rectangle $R$ de surface $2 \pi$ dans le plan temps-fréquence $(t, \xi)$, et qui satisfait, outre la condition de normalisation $\left\|f_{R}\right\|_{L^{2}}=1$, aux exigences de localisation suivantes

$$
\begin{gathered}
\int_{\mathbb{R}}\left(t-t_{0}\right)^{2}\left|f_{R}(t)\right|^{2} d t \leq K^{2} h^{2} \\
\int_{\mathbb{R}}\left(\xi-\omega_{0}\right)^{2}\left|\hat{f}_{R}(\xi)\right|^{2} d \xi \leq \frac{2 \pi K^{2}}{h^{2}}
\end{gathered}
$$

Ici, $\left(t_{0}, \xi_{0}\right)$ est le centre du rectangle $R$, qui se définit comme la région du plan d'équations

$$
t_{0}-h \leq t \leq t_{0}+h, \quad \xi_{0}-\frac{2 \pi}{h} \leq \xi \leq \xi_{0}+\frac{2 \pi}{h} .
$$

$\hat{f}$ désigne la transformée de Fourier de $f$, et $K$ est une constante positive. Si l'on impose à $f_{R}$ d'être à valeurs réelles, il faut modifier la condition (1.2) en remplaçant l'intégrale sur $\mathbb{R}$ par une intégrale sur $\mathbb{R}_{+}$.

Le but de l'analyse temps-fréquence est d'écrire un signal donné comme combinaison linéaire d'atomes temps-fréquence choisis de manière "optimale", suivant un critère qui dépend de l'utilisation de ce signal.

Une façon d'atteindre ce but est de disposer d'une bibliothèque de bases orthonormées d'atomes temps-fréquence. Cette bibliothèque doit être assez vaste pour s'adapter à la grande variété de signaux possibles, mais pas trop, car on veut également disposer d'un algorithme rapide pour sélectionner la base qui représente au mieux un signal. 
Les bases de paquets d'ondelettes constituent une telle bibliothèque, à ceci près que ce ne sont pas toutes des bases d'atomes tempsfréquence. Coifman, Meyer et Wickerhauser [4] ont en effet montré que la bibliothèque contient de mauvaises "pages" (ou paquets d'ondelettes) dans certains de ses "livres" (ou bases). Cependant, tout n'est pas perdu en pratique. En effet, la bibliothèque est générée à l'aide de filtres conjugués en quadrature, et l'on explique dans [4] comment les choisir de façon à réduire les artefacts. Malheureusement, ce choix correspond à des filtres plus longs, d'où un temps de calcul augmenté.

Une autre solution est de n'utiliser, dans la bibliothèque, que les "bons livres". Comme le choix est plus restreint, on gagne en temps de calcul, mais on perd en pertinence de la représentation. Pour appliquer cette solution, il convient de disposer, pour des filtres donnés, d'un "guide" indiquant les qualités de localisation des différents paquets d'ondelettes de la bibliothèque.

Dans le présent travail, nous nous proposons de fournir de tels guides, pour une large classe de filtres conjugués en quadrature. Ces guides présentent une lacune: le critère de localisation fréquentielle utilisé est plus faible que (1.2), et ne peut conduire qu'à une estimation inférieure sur la "variance"

$$
\int_{\mathbb{R}}\left(\xi-\omega_{0}\right)^{2}\left|\hat{f}_{R}(\xi)\right|^{2} d \xi
$$

\section{Enoncé des résultats.}

Dans tout ce qui suit, nous appellerons "couple de filtres conjugués en quadrature" la donnée de deux fonctions à valeurs complexes $\left(m_{0}, m_{1}\right)$ ayant les propriétés suivantes

$m_{0}$ et $m_{1}$ sont de classe $C^{\infty}, 2 \pi$-périodiques

et vérifient $m_{\varepsilon}(-\xi)=\overline{m_{\varepsilon}(\xi)}, \xi \in \mathbb{R}, \varepsilon=1,2$.

Pour tout $\xi$ réel, la matrice $\left(\begin{array}{cc}m_{0}(\xi) & m_{1}(\xi) \\ m_{0}(\xi+\pi) & m_{1}(\xi+\pi)\end{array}\right)$

est unitaire.

$m_{0}(0)=1 \quad$ et $\quad m_{0}(\xi) \neq 0 \quad$ pour tout $\xi \in\left[-\frac{\pi}{2}, \frac{\pi}{2}\right]$. 
En notant $M_{\varepsilon}(\xi)=\left|m_{\varepsilon}(\xi)\right|, \varepsilon=1,2$, il résulte de (2.1), (2.2) et (2.3), que $M_{\varepsilon}$ est une fonction paire, $2 \pi$-périodique, Lipschitzienne, avec $M_{1}(\xi)=M_{0}(\xi+\pi)$ et $M_{0}(\xi)^{2}+M_{0}(\xi+\pi)^{2}=1$.

La fonction somme $S(\xi)=M_{0}(\xi)+M_{0}(\xi+\pi)$ est donc paire, $\pi$-périodique, Lipschitzienne, à valeurs dans $[1, \sqrt{2}]$, avec $S(0)=1$, $S(\pi / 2)=\sqrt{2}$.

Les propriétés (2.1) et (2.3) entraînent (voir [1]) que le produit $\prod_{p=1}^{\infty} m_{0}\left(\xi / 2^{p}\right)$ converge pour presque tout $\xi$ réel, et qu'il existe une constante $\lambda>0$ telle que

$$
\left|\prod_{p=1}^{\infty} m_{0}\left(\xi / 2^{p}\right)\right| \geq \lambda, \quad \text { pour tout } \xi \text { dans }[-\pi, \pi] .
$$

Ce produit est la transformée de Fourier $\hat{\varphi}(\xi)$ de la "fonction d'échelle" $\varphi(t)$.

Les paquets d'ondelettes sont des fonctions $w_{n}, n \geq 0$, définies par leur transformée de Fourier

$$
\hat{w}_{n}(\xi)=m_{\varepsilon_{1}}(\xi / 2) \cdots m_{\varepsilon_{j}}\left(\xi / 2^{j}\right) \hat{\varphi}\left(\xi / 2^{j}\right),
$$

pour $n=\sum_{q=1}^{j} \varepsilon_{q} 2^{q-1}$.

On a bien sûr $w_{0}=\varphi$, et pour $2^{j-1} \leq n<2^{j}, w_{n}$ est combinaison linéaire de fonctions du type $\psi\left(2^{j-1} t-k\right), k \in \mathbb{Z}$, où $\psi=w_{1}$ est l'ondelette associée au couple de filtres conjugués en quadrature.

Il résulte de $(2.2)$ que les fonctions $w_{n}$ sont normalisées dans $L^{2}(\mathbb{R})$ et deux à deux orthogonales. La bibliothèque de bases orthonormées de $L^{2}(\mathbb{R})$ associée à ces fonctions est indexée par les partitions de $\mathbb{R}_{+}$ en intervalles dyadiques, c'est-à-dire du type $\left[n / 2^{p},(n+1) / 2^{p}\right], n \in \mathbb{N}$, $p \in \mathbb{Z}$.

La base ayant pour indice une telle partition $\left(I_{\alpha}\right)_{\alpha \in A}$, est la collection de fonctions

$$
\left(2^{-p_{\alpha} / 2} w_{n_{\alpha}}\left(2^{-p_{\alpha}} t-k\right)\right)_{\alpha \in A, k \in \mathbb{Z}} \quad, \quad \text { avec } \quad I_{\alpha}=\left[\frac{n_{\alpha}}{2^{p_{\alpha}}}, \frac{n_{\alpha}+1}{2^{p_{\alpha}}}\right] .
$$

Si l'on remplace $\left(m_{0}, m_{1}\right)$ par les filtres "parfaits"

$$
\chi_{0}=1_{[-\pi / 2, \pi / 2]+2 \pi \mathbb{Z}} \text { et } \quad \chi_{1}=1_{[\pi / 2,3 \pi / 2]+2 \pi \mathbb{Z}},
$$


$1_{X}$ étant la fonction caractéristique de $X$, on trouve

$$
\hat{w}_{n}(\xi)=1_{J \cup(-J)}(\xi),
$$

pour $n=\sum_{q \geq 1} \varepsilon_{q} 2^{q-1}, J=\pi[\mathcal{G}(n), \mathcal{G}(n)+1], \mathcal{G}(n)=\sum_{q \geq 1} \gamma_{q} 2^{q-1}$, $\gamma_{q}=\left|\varepsilon_{q+1}-\varepsilon_{q}\right|$. On appelle "code de Gray" la correspondance

$$
\left(\varepsilon_{q}\right) \rightarrow\left(\gamma_{q}\right)
$$

Cette formule suggère que $w_{n}$ est un atome temps-fréquence associé au rectangle $R$ d'équations $-1 \leq t \leq 1, \pi \mathcal{G}(n) \leq \xi \leq \pi(\mathcal{G}(n)+1)$.

Dans le cas des filtres parfaits, la condition (1.1) ne peut être satisfaite par aucun des $w_{n}$. A l'opposé, si l'on travaille avec des filtres $\left(m_{0}, m_{1}\right)$ qui sont des polynômes trigonométriques, alors $w_{n}(t)$ est à support inclus dans un compact indépendant de $n$, et (1.1) est vérifiée. Nous ne savons pas si (1.1) est encore vraie pour des filtres conjugués en quadrature plus généraux, sous les seules hypothèses (2.1), (2.2), (2.3).

Pour étudier la condition (1.2), on part de l'inégalité suivante (on rappelle que $w_{n}$ est normalisée au sens $L^{2}$ ):

$$
\int_{-\infty}^{+\infty}\left|\hat{w}_{n}(\xi)\right| d \xi \leq \pi\left(2+\sigma_{n}\right)^{1 / 2}
$$

avec

$$
\sigma_{n}=\frac{1}{2 \pi} \inf _{\omega_{0} \geq 0} \int_{0}^{+\infty}\left|\xi-\omega_{0}\right|^{2}\left|\hat{w}_{n}(\xi)\right|^{2} d \xi
$$

D'après (2.5), une minoration sur $\left\|\hat{w}_{n}\right\|_{L^{1}(\mathbb{R})}$ donne une minoration sur la localisation de $\hat{w}_{n}$ au sens de (1.2). En revanche, une majoration de $\left\|\hat{w}_{n}\right\|_{L^{1}(\mathbb{R})}$ ne donne aucun renseignement sur $\sigma_{n}$. Cependant, dans tout ce qui va suivre, on étudiera uniquement $\left\|\hat{w}_{n}\right\|_{L^{1}(\mathbb{R})}$, qui est plus facile à manipuler que $\sigma_{n}$ (cette démarche est la même que dans [4]).

Etant donné un couple $\left(m_{0}, m_{1}\right)$ de filtres conjugués en quadrature, il est prouvé dans [4] qu'il existe une constante $\rho>1$ telle que pour tout $j \geq 0$, on ait

$$
\frac{1}{2^{j}} \sum_{n=0}^{2^{j}-1}\left\|\hat{w}_{n}\right\|_{L^{1}} \geq 2 \pi \rho^{j}
$$


En d'autres termes, toutes les fonctions $w_{n}$ ne sont pas des atomes temps-fréquence.

Dans l'hypothèse où $\left|m_{0}\right|$ vaut 1 sur $[-\pi / 3, \pi / 3]$, on trouve dans [4] l'estimation suivante:

$$
\left\|\hat{w}_{n}\right\|_{L^{1}} \leq C n^{1 / 4}
$$

Si l'on recherche une croissance plus lente de $\left\|\hat{w}_{n}\right\|_{L^{1}}$, il est naturel de choisir $m_{0}$ proche du filtre "parfait" $\chi_{0}$. Plus précisément (voir [4]), si $\left|m_{0}\right|$ vaut 1 sur l'intervalle $[-\pi / 2+\delta, \pi / 2-\delta]$ avec $0<\delta \leq \pi / 6$, alors

$$
\left\|\hat{w}_{n}\right\|_{L^{1}} \leq C(\delta) n^{\gamma(\delta)} \text { et }(C \rightarrow 2 \pi, \gamma \rightarrow 0) \text { lorsque } \delta \rightarrow 0 .
$$

Remarquons que la minoration (2.7) n'interdit pas l'existence d'une suite $\left(n_{p}\right)_{p \geq 0}$ strictement croissante telle que $\left\|\hat{w}_{n_{p}}\right\|_{L^{1}}$ reste bornée. Les résultats que nous allons énoncer puis démontrer sont des encadrements de $\left\|\hat{w}_{n}\right\|_{L^{1}}$ en fonction du développement dyadique de $n$. Les formules obtenues dépendront d'hypothèses sur $\left(m_{0}, m_{1}\right)$. Nous appellerons $\left(H_{p}\right)_{p \geq 1}$ les hypothèses donnant les majorations, et $\left(h_{p}\right)_{p \geq 1}$ celles qui donneront les minorations.

L'hypothèse $\left(H_{1}\right)$ assure que $\left\|\hat{w}_{0}(\xi)\right\|_{L^{1}(\mathbb{R})}$ est finie. Elle est immédiatement vérifiée lorsque $M_{0}=1$ sur $[-\pi / 3, \pi / 3]$. Elle est vraie aussi pour les filtres d'Ingrid Daubechies $m_{0}^{N}, N \geq 2$ (voir [1]).

Hypothèse $\left(H_{1}\right)$. On peut écrire

$$
m_{0}(\xi)=\left(\frac{1+e^{i \xi}}{2}\right)^{N} B(\xi)
$$

et si l'on pose

$$
b_{j}=\frac{1}{j \log 2} \sup _{\xi \in \mathbb{R}} \sum_{k=1}^{j} \log \left|B\left(2^{k} \xi\right)\right|,
$$

on a l'inégalité

$$
b=\inf _{j \geq 1} b_{j}<N-1
$$


Si l'hypothèse $\left(H_{1}\right)$ est vraie (voir [1]), alors pour $\varepsilon>0$ assez petit, il existe une constante $K_{\varepsilon}>0$ telle que pour tout $\xi$ réel,

$$
|\hat{\varphi}(\xi)| \leq \frac{K_{\varepsilon}}{(1+|\omega|)^{1+\varepsilon}}
$$

Nous démontrerons le résultat suivant au Section IV:

Théorème $\left(H_{1}\right)$. Supposons l'hypothèse $\left(H_{1}\right)$ vérifiée. Avec les notations

$$
\begin{gathered}
C=\|\hat{\varphi}\|_{L^{1}(\mathbb{R})}, \quad R=\sup _{\xi \in[0, \pi]} \sum_{k \in \mathbb{Z}}|\hat{\psi}(\xi-2 k \pi)|, \\
N_{1}(n)=\sum_{q \geq 1} \varepsilon_{q}, \quad n=\sum_{q \geq 1} \varepsilon_{q} 2^{q-1}
\end{gathered}
$$

on a l'inégalité

$$
\left\|\hat{w}_{n}\right\|_{L^{1}(\mathbb{R})} \leq C R^{N_{1}(n)}
$$

L'hypothèse $\left(h_{1}\right)$ permettra d'obtenir une estimation inverse (on rappelle que $\left.M_{0}=\left|m_{0}\right|, S(\xi)=M_{0}(\xi)+M_{0}(\xi+\pi)\right)$.

Hypothèse $\left(h_{1}\right)$. Il existe $\alpha>1$ tel que pour tout $\xi \in[\pi / 4, \pi / 2]$, on ait $S(\xi) \geq \alpha$. De plus, $M_{0}(\xi) \geq 1 / \sqrt{2} \operatorname{sur}[-\pi / 2, \pi / 2]$.

Cette estimation est la suivante:

Théorème $\left(h_{1}\right)$. Si l'hypothèse $\left(h_{1}\right)$ est vérifiée, en posant

$$
r=\frac{\alpha+1}{2}
$$

on a l'inégalité

$$
\left\|\hat{w}_{n}\right\|_{L^{1}(\mathbb{R})} \geq 2 \pi r^{N_{1}(n)},
$$

$N_{1}$ étant défini comme dans le Théorème $\left(H_{1}\right)$.

Les deux résultats qui précèdent peuvent s'appliquer aux filtres de longueur finie: 
Corollaire 1. Si $m_{0}$ est un filtre d'Ingrid Daubechies $m_{0}^{N}$ avec $N \geq 2$, il existe $R>r>1$ et $C>0$ tels que pour tout $n \geq 0$, on ait

$$
2 \pi r^{N_{1}(n)} \leq\left\|\hat{w}_{n}\right\|_{L^{1}(\mathbb{R})} \leq C R^{N_{1}(n)},
$$

$N_{1}$ étant défini comme dans le Théorème $\left(H_{1}\right)$.

Remarque. Pour $N$ grand, le Théorème $\left(h_{1}\right)$ donne une valeur de $r$ très proche de 1 . En revanche, la valeur de $R$ donnée par le Théorème $\left(H_{1}\right)$ n'est jamais inférieure à $\sqrt{2}$, comme le montre le calcul suivant:

$$
\begin{aligned}
R & \geq \sum_{k \in \mathbb{Z}}|\hat{\psi}(\pi-2 k \pi)| \\
& =M_{1}\left(\frac{\pi}{2}\right) \sum_{l \in \mathbb{Z}}\left|\hat{\varphi}\left(\frac{\pi}{2}-2 l \pi\right)\right|+M_{0}\left(\frac{\pi}{2}\right) \sum_{k \in \mathbb{Z}}\left|\hat{\varphi}\left(\frac{\pi}{2}-2 l \pi-\pi\right)\right| \\
& =\sqrt{2} \sum_{l \in \mathbb{Z}}\left|\hat{\varphi}\left(\frac{\pi}{2}-2 l \pi\right)\right| \\
& \geq \sqrt{2}\left|\sum_{l \in \mathbb{Z}} \hat{\varphi}\left(\frac{\pi}{2}-2 l \pi\right)\right|=\sqrt{2} .
\end{aligned}
$$

Le Corollaire 1 donne donc une estimation peu précise. Cependant, cette estimation est suffisante lorsqu'il s'agit de savoir si une sous-suite $\left\|\hat{w}_{n_{p}}\right\|_{L^{1}}$ est bornée ou non.

Les hypothèses $\left(H_{p}\right)$ et $\left(h_{p}\right)$ pour $p \geq 2$ concernent des filtres tels que $M_{0}=1$ sur $[-\pi / 3, \pi / 3]$.

Hypothèse $\left(H_{p}\right)$. Pour $\delta=\delta_{p}=\pi /\left(2\left(2^{p}-1\right)\right), \quad M_{0}$ vaut 1 sur

$$
\left[-\frac{\pi}{2}+\delta, \frac{\pi}{2}-\delta\right]
$$

et il existe $\beta<1$ tel que

$$
M_{0}(\xi) \leq \beta \frac{\left(2^{p}-1\right)^{2}}{\pi}\left(\xi-\frac{\pi}{2}+\delta\right) \quad \text { sur }\left[\frac{\pi}{2}-\delta, \frac{\pi}{2}+\delta\right] .
$$

Hypothèse $\left(h_{p}\right) . M_{0}$ vaut 1 sur $[-\pi / 3, \pi / 3]$, et il existe $\alpha>1$ tel que

$$
S(\xi) \geq \alpha \quad \text { pour } \xi \in\left[\frac{\pi}{2}\left(1-\frac{1}{2^{p}}\right), \frac{\pi}{2}\right] .
$$

De plus, $M_{0}(\xi) \geq 1 / \sqrt{2}$ sur $[-\pi / 2, \pi / 2]$. 
Ces hypothèses donnent deux estimations inverses l'une de l'autre:

Théorème $\left(H_{p}\right)$. Si l'hypothèse $\left(H_{p}\right)$ est vérifiée, il existe deux constantes $C_{p}>2 \pi$ et $R=R(\beta, p)>1$ telles que pour tout $n \geq 0$, on ait

$$
\left\|\hat{w}_{n}\right\|_{L^{1}(\mathbb{R})} \leq C_{p} R^{N_{p}(n)+1}
$$

Ici, $N_{p}(n)$ est le nombre de fois qu'apparaît la suite

$$
\underbrace{0 \ldots 0}_{(p-1) \text { fois } 0} 1
$$

dans le développement dyadique $\left(\varepsilon_{1} \ldots \varepsilon_{j} \ldots\right)$ de $n=\sum_{q \geq 1} \varepsilon_{q} 2^{q-1}$. De plus, on peut prendre

$$
C_{p}=2 \pi+4 \delta_{p}(\sqrt{2}-1) \quad \text { et } \quad R(\beta, p)=3+\frac{2^{p+1} \beta}{1-\beta} .
$$

Théorème $\left(h_{p}\right)$. Si l'hypothèse $\left(h_{p}\right)$ est vérifiée, en posant

$$
r=\frac{\alpha+1}{2},
$$

on a l'inégalité

$$
\left\|\hat{w}_{n}\right\|_{L^{1}(\mathbb{R})} \geq 2 \pi r^{N_{p}(n)},
$$

$N_{p}$ étant le même que dans le Théorème $\left(H_{p}\right)$.

Remarquons que pour tout $p \geq 2,\left(H_{p}\right)$ implique $\left(H_{p-1}\right),\left(h_{p}\right)$ implique $\left(h_{p+1}\right)$, et si $\left(h_{p}\right)$ est vraie, alors $\left(H_{p+1}\right)$ est fausse.

Enfin, si un couple de filtres conjugués en quadrature est tel que $M_{0}=1$ sur $[-\pi / 3, \pi / 3]$ et que $M_{0}$ soit décroissante sur $[0, \pi / 2]$, alors il existe $p \geq 2$ tel que $\left(h_{p}\right)$ et $\left(H_{p-1}\right)$ soient simultanément satisfaites par ce couple, $\left(H_{p}\right)$ pouvant être ou non vérifiée.

Tout ceci se voit immédiatement à partir des énoncés des hypothèses, et on a le résultat suivant:

Corollaire 2. Supposons que $M_{0}=1$ sur $[-\pi / 3, \pi / 3]$ avec $M_{0}$ décroissante sur $[0, \pi / 2]$. Soit $p$ le plus petit entier tel que

$$
M_{0}\left(\frac{\pi}{2}\left(1-\frac{1}{2^{p}}\right)\right)<1
$$


Il existe $R>r>1$ et $C>0$ tels que pour tout $n \geq 0$, on ait

$$
2 \pi r^{N_{p}(n)} \leq\left\|\hat{w}_{n}\right\|_{L^{1}(\mathbb{R})} \leq C R^{N_{p-1}(n)},
$$

$N_{p}$ étant défini comme dans le Théorème $\left(H_{p}\right)$.

En pratique, les algorithmes de paquets d'ondelettes sont utilisés avec des filtres à réponse impulsionnelle finie. Pour de tels filtres, on n'a pas $M_{0}=1$ sur $[-\pi / 3, \pi / 3]$, et seul le Corollaire 1 s'applique. Cependant, pour des filtres assez longs, on peut avoir $M_{0}$ très proche de 1 sur $[-\pi / 3, \pi / 3]$, ce qui rend peu précis l'encadrement du Corollaire 1 (voir la remarque plus haut). Dans ce cas, le Corollaire 2 pourrait donner des indications sur le comportement de $\left\|\hat{w}_{n}\right\|_{L^{1}(\mathbb{R})}$. Nous n'étudierons pas cette question plus avant.

Par ailleurs, il existe un algorithme de décomposition adaptative en fonctions cosinus locales, imaginé par R.R. Coifman, et appelé algorithme de repliement multiple (voir [5]). Les bases générées par cet algorithme sont les transformées de Fourier des bases de paquets d'ondelettes, et les filtres $\left(m_{0}, m_{1}\right)$ associés vérifient les hypothèses du Corollaire 2.

Signalons enfin que les estimations (2.7) et (2.9), obtenues par une méthode directe dans [4], peuvent facilement être déduites des Théorèmes $\left(h_{p}\right)$ et $\left(H_{p}\right)$.

\section{Démonstration des Théorèmes $\left(h_{p}\right)$.}

On rappelle les notations $M_{\varepsilon}=\left|m_{\varepsilon}\right|, S=M_{0}+M_{1}$.

Soit $n=\sum_{q \geq 1} \varepsilon_{q} 2^{q-1}$. On pose $W_{n}^{0}=1$, et pour $j \geq 1$,

$$
W_{n}^{j}(\xi)=M_{\varepsilon_{1}}(\xi / 2) \cdots M_{\varepsilon_{j}}\left(\xi / 2^{j}\right) .
$$

Posons également $n_{j}=\sum_{q=1}^{j} \varepsilon_{q} 2^{q-1}$. Si $j \geq \log _{2}(n)$, alors $n_{j}=n$, et d'après (2.4),

$$
W_{n}^{j}(\xi) \leq \frac{\left|\hat{w}_{n}(\xi)\right|}{\lambda} \quad \text { sur }\left[-2^{j} \pi, 2^{j} \pi\right]
$$

Donc, par le théorème de convergence dominée de Lebesgue,

$$
\left\|\hat{w}_{n}(\xi)\right\|_{L^{1}(\mathbb{R})}=\lim _{j \rightarrow+\infty}\left\|W_{n}^{j}(\xi)\right\|_{L^{1}\left(-2^{j} \pi, 2^{j} \pi\right)} \cdot
$$


De plus, on dispose de la propriété de monotonie suivante:

Lemme 1. Soit $\left(m_{0}, m_{1}\right)$ un couple de filtres conjugués en quadrature, et soit $\left(\varepsilon_{1}, \ldots, \varepsilon_{j}\right)$ une suite dans $\{0,1\}^{j}$. On a

$$
\begin{aligned}
2^{j} \| m_{\varepsilon_{j}}(\xi) m_{\varepsilon_{j-1}}(2 \xi) \cdots & m_{\varepsilon_{1}}\left(2^{j} \xi\right) \|_{L^{1}(0,2 \pi)} \\
& \geq 2^{j-1}\left\|m_{\varepsilon_{j-1}}(\xi) \cdots m_{\varepsilon_{1}}\left(2^{j-1} \xi\right)\right\|_{L^{1}(0,2 \pi)} .
\end{aligned}
$$

Pour $n$ fixé, la suite $\left\|W_{n}^{j}(\xi)\right\|_{L^{1}\left(-2^{j} \pi, 2^{j} \pi\right)}$ est donc croissante, et

$$
\left\|\hat{w}_{n}(\xi)\right\|_{L^{1}(\mathbb{R})}=\sup _{j \geq 0}\left\|W_{n}^{j}(\xi)\right\|_{L^{1}\left(-2^{j} \pi, 2^{j} \pi\right)} \cdot
$$

DÉmonstration. On pose $F(\xi)=2^{j-1}\left|m_{\varepsilon_{j-1}}(\xi) \cdots m_{\varepsilon_{1}}\left(2^{j-1} \xi\right)\right| \cdot F$ est continue, $2 \pi$-périodique, paire et à valeurs positives ou nulles. On calcule

$$
\begin{aligned}
2 \int_{0}^{2 \pi}\left|m_{\varepsilon_{j}}(\xi)\right| F(2 \xi) d \xi & =2 \int_{0}^{2 \pi} M_{0}(\xi) F(2 \xi) d \xi \\
& =\int_{0}^{2 \pi} S\left(\frac{\xi}{2}\right) F(\xi) d \xi
\end{aligned}
$$

en rappelant que $S(x)=M_{0}(x)+M_{0}(x+\pi)$. Comme on a toujours $1 \leq S(x) \leq \sqrt{2}$, le Lemme 1 est démontré.

Nous allons démontrer les Théorèmes $\left(h_{p}\right)$ en minorant les normes $\left\|W_{n}^{j}(\xi)\right\|_{L^{1}\left(-2^{j} \pi, 2^{j} \pi\right)}$ à l'aide d'une récurrence sur $j$, pour $n$ fixé. Il est clair que $\left\|W_{n}^{0}(\xi)\right\|_{L^{1}(-\pi, \pi)}=2 \pi$. faite.

Supposons dans un premier temps que l'hypothèse $\left(h_{1}\right)$ est satis-

Si $\varepsilon_{j}=0$, alors $N_{1}\left(n_{j}\right)=N_{1}\left(n_{j-1}\right)$, et par le Lemme 1,

$$
\left\|W_{n}^{j+1}(\xi)\right\|_{L^{1}\left(-2^{j+1} \pi, 2^{j+1} \pi\right)} \geq\left\|W_{n}^{j}(\xi)\right\|_{L^{1}\left(-2^{j} \pi, 2^{j} \pi\right)} .
$$

Si $\varepsilon_{j}=1$, alors $N_{1}\left(n_{j}\right)=N_{1}\left(n_{j-1}\right)+1$. De plus, le quotient de $\left\|W_{n}^{j+1}(\xi)\right\|_{L^{1}\left(-2^{j+1} \pi, 2^{j+1} \pi\right)}$ par $\left\|W_{n}^{j}(\xi)\right\|_{L^{1}\left(-2^{j} \pi, 2^{j} \pi\right)}$ est égal au quotient de $2 \int_{-\pi}^{\pi} M_{\varepsilon_{j+1}}(\xi) M_{1}(2 \xi) f(4 \xi) d \xi$ par $\int_{-\pi}^{\pi} M_{1}(\xi) f(2 \xi) d \xi$, avec la notation $f(\xi)=W_{n}^{j-1}(\xi)$. 
$f$ est continue, $2 \pi$-périodique, paire et à valeurs positives ou nulles, et on trouve

$$
\begin{aligned}
& 2 \int_{-\pi}^{\pi} M_{\varepsilon_{j+1}}(\xi) M_{1}(2 \xi) f(4 \xi) d \xi \\
& \quad= \frac{1}{2} \int_{-\pi}^{\pi} f(\xi)\left[S\left(\frac{\xi}{4}\right) M_{1}\left(\frac{\xi}{2}\right)+S\left(\frac{\xi}{4}+\frac{\pi}{2}\right) M_{1}\left(\frac{\xi}{2}+\pi\right)\right] d \xi \\
& \quad \geq \frac{1}{2} \int_{-\pi}^{\pi} f(\xi)\left(M_{1}\left(\frac{\xi}{2}\right)+\alpha M_{1}\left(\frac{\xi}{2}+\pi\right)\right) d \xi
\end{aligned}
$$

Or, d'après l'hypothèse $\left(h_{1}\right)$, on a $M_{1}(\xi / 2) \leq 1 / \sqrt{2} \leq M_{1}(\xi / 2+\pi)$ pour tout $|\xi| \leq \pi$. D'autre part, quels que soient $x \leq y$ et $a \leq b$, on a l'inégalité $x a+y b \geq(x+y)(a+b) / 2$. Si l'on prend $x=1, y=\alpha$, $a=M_{1}(\xi / 2), b=M_{1}(\xi / 2+\pi)$, on en déduit

$$
\begin{aligned}
2 \int_{-\pi}^{\pi} M_{\varepsilon_{j+1}}(\xi) M_{1}(2 \xi) f(4 \xi) d \xi & \geq \frac{1+\alpha}{4} \int_{-\pi}^{\pi} f(\xi) S\left(\frac{\xi}{2}\right) d \xi \\
& =\frac{1+\alpha}{2} \int_{-\pi}^{\pi} M_{1}(\xi) f(2 \xi) d \xi
\end{aligned}
$$

Si l'on pose $r=(1+\alpha) / 2$, on vient de démontrer par récurrence sur $j$ que

$$
\left\|W_{n}^{j}(\xi)\right\|_{L^{1}\left(-2^{j} \pi, 2^{j} \pi\right)} \geq 2 \pi r^{N_{1}\left(n_{j-1}\right)} .
$$

En prenant $j>\log _{2} n$, on déduit le Théorème $\left(h_{1}\right)$ de (3.4) et (3.5).

Plus généralement, pour montrer le Théorème $\left(h_{p}\right)$, il suffit de prouver que le quotient de l'intégrale

$$
A=2 \int_{-\pi}^{\pi} f\left(2^{p+1} \xi\right) M_{\varepsilon}(\xi) M_{1}(2 \xi) \prod_{q=1}^{p-1} M_{0}\left(2^{q+1} \xi\right) d \xi
$$

par l'intégrale

$$
B=\int_{-\pi}^{\pi} f\left(2^{p} \xi\right) M_{1}(\xi) \prod_{q=1}^{p-1} M_{0}\left(2^{q} \xi\right) d \xi
$$

est supérieur ou égal à $r=(1+\alpha) / 2$, pour toute fonction $f$ continue, $2 \pi$-périodique, paire et à valeurs positives ou nulles, et tout $\varepsilon \in\{0,1\}$. 
Pour cela, on commence par vérifier, par récurrence sur $j$, que le produit $M_{0}(\xi) \cdots M_{0}\left(2^{j-1} \xi\right) f\left(2^{j} \xi\right)$ vaut $M_{0}\left(2^{j-1} \xi\right) f\left(2^{j} \xi\right)$ pour $\xi$ dans $\left[-\pi /\left(3 \cdot 2^{j-2}\right), \pi /\left(3 \cdot 2^{j-2}\right)\right]+2 \pi \mathbb{Z}$ et vaut 0 partout ailleurs.

On en déduit que

$$
M_{1}(\xi) \prod_{q=1}^{p-1} M_{0}\left(2^{q} \xi\right) f\left(2^{p} \xi\right)=M_{0}\left(2^{p-1} \xi\right) f\left(2^{p} \xi\right)
$$

sur

$$
\left[\pi\left(1-\frac{1}{3 \cdot 2^{p-2}}\right), \pi\left(1+\frac{1}{3 \cdot 2^{p-2}}\right)\right]+2 \pi \mathbb{Z},
$$

et vaut 0 partout ailleurs. Donc

$$
\begin{aligned}
A & =4 \int_{\pi\left(1-1 /\left(3 \cdot 2^{p-2}\right)\right) / 2}^{\pi\left(1+1 /\left(3 \cdot 2^{p-2}\right)\right) / 2} M_{0}(\xi) M_{0}\left(2^{p} \xi\right) f\left(2^{p+1} \xi\right) d \xi \\
& =2 \int_{\pi}^{\pi\left(1+1 /\left(3 \cdot 2^{p-2}\right)\right)} S\left(\frac{\xi}{2}\right) M_{0}\left(2^{p-1} \xi\right) f\left(2^{p} \xi\right) d \xi .
\end{aligned}
$$

On découpe maintenant l'intervalle $I=\left[\pi, \pi\left(1+1 /\left(3 \cdot 2^{p-2}\right)\right)\right]$ en trois parties

$$
\begin{aligned}
& I_{1}=\left[\pi, \pi\left(1+\frac{1}{3 \cdot 2^{p-1}}\right)\right], \\
& I_{2}=\left[\pi\left(1+\frac{1}{3 \cdot 2^{p-1}}\right), \pi\left(1+\frac{1}{2^{p}}\right)\right] \\
& I_{3}=\left[\pi\left(1+\frac{1}{2^{p}}\right), \pi\left(1+\frac{1}{3 \cdot 2^{p-2}}\right)\right] .
\end{aligned}
$$

Sur $I_{1} \cup I_{2}$, on a $S(\xi / 2) \geq \alpha$, et $I_{3}$ est le symétrique de $I_{2}$ par rapport à $\pi\left(1+1 / 2^{p}\right)$. Donc

$$
\int_{I_{1}} S\left(\frac{\xi}{2}\right) M_{0}\left(2^{p-1} \xi\right) f\left(2^{p} \xi\right) d \xi \geq \alpha \int_{I_{1}} M_{0}\left(2^{p-1} \xi\right) f\left(2^{p} \xi\right) d \xi
$$

et

$$
\begin{array}{rl}
\int_{I_{2} \cup I_{3}} & S\left(\frac{\xi}{2}\right) M_{0}\left(2^{p-1} \xi\right) f\left(2^{p} \xi\right) d \xi \\
& =\int_{I_{2}} f\left(2^{p} \xi\right)\left(S\left(\frac{\xi}{2}\right) M_{0}\left(2^{p-1} \xi\right)\right.
\end{array}
$$




$$
\begin{aligned}
& \left.+S\left(2 \pi\left(1+\frac{1}{2^{p}}\right)-\frac{\xi}{2}\right) M_{0}\left(2^{p-1} \xi+\pi\right)\right) d \xi \\
\geq & \int_{I_{2}}\left(\alpha M_{0}\left(2^{p-1} \xi\right)+1 M_{0}\left(2^{p-1} \xi+\pi\right)\right) f\left(2^{p} \xi\right) d \xi \\
\geq & \frac{1+\alpha}{2} \int_{I_{2}} S\left(2^{p-1} \xi\right) f\left(2^{p} \xi\right) d \xi \\
= & \frac{1+\alpha}{2} \int_{I_{1} \cup I_{2}} M_{0}\left(2^{p-1} \xi\right) f\left(2^{p} \xi\right) d \xi .
\end{aligned}
$$

Finalement, on trouve l'inégalité cherchée,

$$
A \geq \frac{1+\alpha}{2} \int_{\pi}^{\pi\left(1+1 /\left(3 \cdot 2^{p-2}\right)\right)} 2 M_{0}\left(2^{p-1} \xi\right) f\left(2^{p} \xi\right) d \xi=r B .
$$

On en déduit, par récurrence sur $j$, que

$$
\left\|W_{n}^{j}(\xi)\right\|_{L^{1}\left(-2^{j} \pi, 2^{j} \pi\right)} \geq 2 \pi r^{N_{p}\left(n_{j-1}\right)} .
$$

En prenant $j>\log _{2} n$, on déduit le Théorème $\left(h_{p}\right)$ de $(3.4)$ et (3.6).

\section{Démonstration des Théorèmes $\left(H_{p}\right)$.}

On rappelle les notations

$$
M_{\varepsilon}=\left|m_{\varepsilon}\right|, \quad S=M_{0}+M_{1}, \quad W_{n}^{j}(\xi)=M_{\varepsilon_{1}}(\xi / 2) \cdots M_{\varepsilon_{j}}\left(\xi / 2^{j}\right) .
$$

D'après (3.3), il suffit, pour obtenir une majoration de $\left\|\hat{w}_{n}(\xi)\right\|_{L^{1}(\mathbb{R})}$, de majorer $\left\|W_{n}^{j}(\xi)\right\|_{L^{1}\left(-2^{j} \pi, 2^{j} \pi\right)}$ indépendamment de $j$.

Si l'hypothèse $(H 1)$ est satisfaite, en posant

$$
s^{\infty}(\xi)=\sum_{k \in \mathbb{Z}}|\hat{\psi}(\xi-2 k \pi)|
$$

il résulte de la formule $(2.10)$ que $R=\sup _{\xi \in[0, \pi]} s^{\infty}(\xi)<+\infty$. On note

$$
s^{m}(\xi)=\sum_{k=0}^{2^{m}-1} W_{1}^{m}(\xi+2 k \pi)
$$


D'après (2.4) et la formule de convergence dominée de Lebesgue, $s^{m}$ converge simplement vers $s^{\infty}$ lorsque $m$ tend vers l'infini. De plus, on a

$$
s^{m+1}(\xi)=s^{m}(\xi) S\left(2^{-m-1} \xi\right) \geq s^{m}(\xi) .
$$

Par conséquent,

$$
s^{m}(\xi) \leq s^{\infty}(\xi) \leq R
$$

Par ailleurs, pour $m \geq j>\log _{2} n$, on a l'identité

$$
W_{n+2^{j}}^{m}(\xi)=W_{n}^{j}(\xi) W_{1}^{m-j}\left(\xi / 2^{j}\right)
$$

On en tire

$$
\begin{aligned}
\left\|W_{n+2^{j}}^{m}\right\|_{L^{1}\left(-2^{m} \pi, 2^{m} \pi\right)} & =\int_{-2^{m} \pi}^{2^{m} \pi} W_{n}^{j}(\xi) W_{1}^{m-j}\left(\xi / 2^{j}\right) d \xi \\
& =\int_{-2^{j} \pi}^{2^{j} \pi} W_{n}^{j}(\xi) s^{m-j}\left(2^{-j} \xi\right) d \xi \\
& \leq R\left\|W_{n}^{j}(\xi)\right\|_{L^{1}\left(-2^{j} \pi, 2^{j} \pi\right)} .
\end{aligned}
$$

Donc, pour $n=2^{j_{1}}+\cdots+2^{j_{N}}, j_{1}<\cdots<j_{N}$, on a

$$
\begin{aligned}
\left\|W_{n}^{m}\right\|_{L^{1}\left(-2^{m} \pi, 2^{m} \pi\right)} & \leq R^{N}\left\|W_{0}^{j_{1}}(\xi)\right\|_{L^{1}\left(-2^{j_{1}} \pi, 2^{j_{1}} \pi\right)} \\
& \leq\|\hat{\varphi}(\xi)\|_{L^{1}(\mathbb{R})} R^{N}=C R^{N} .
\end{aligned}
$$

En faisant tendre $m$ vers l'infini, on obtient le Théorème $\left(H_{1}\right)$ comme conséquence de (3.3) et (4.5).

Supposons maintenant l'hypothèse $\left(H_{2}\right)$ vérifiée.

Soit $f$ une fonction mesurable, positive, paire, $2 \pi$-périodique. Pour avoir l'inégalité stricte

$$
2 \int_{-\pi}^{\pi} M_{\varepsilon}(\xi) f(2 \xi) d \xi>\int_{-\pi}^{\pi} f(\xi) d \xi
$$

il faut que $f$ ait une intégrale non nulle sur $[2 \pi / 3,4 \pi / 3]$.

Donc, s'il existe $g$, elle aussi positive, paire et $2 \pi$-périodique, telle que $f(\xi)=M_{\varepsilon^{\prime}}(\xi) g(2 \xi)$, l'inégalité (4.6) n'est possible que pour $\varepsilon^{\prime}=1$. 
Par conséquent, si l'on pose $h(\xi)=M_{\varepsilon_{j}}(\xi) \cdots M_{\varepsilon_{1}}\left(2^{j-1} \xi\right)$, on a

$$
\int_{-\pi}^{\pi} 2^{m} M_{0}(\xi) \cdots M_{0}\left(2^{m} \xi\right) h\left(2^{m+1} \xi\right) d \xi=\int_{-\pi}^{\pi} M_{0}(\xi) h(2 \xi) d \xi
$$

Si l'on admet l'existence d'une constante $R(\beta)>1$ tel que pour tout $j \geq 1$, en posant

$$
\Sigma_{j}(\xi)=\sum_{k=0}^{2^{j+1}-1} W_{2^{j}-1}^{j+1}(\xi+2 k \pi)
$$

on ait

$$
\sup _{\xi \in[0, \pi]} \Sigma_{j}(\xi) \leq R(\beta)
$$

alors, par des calculs analogues à (4.3), (4.4) et (4.5), on peut majorer $\left\|W_{n}^{m}\right\|_{L^{1}\left(-2^{m} \pi, 2^{m} \pi\right)}$ par $C R^{N_{2}(n)+1}$, avec

$$
C=2 \int_{-\pi}^{\pi} M_{0}(\xi) d \xi, \quad 2 \pi<C<\frac{2 \pi}{3}(2+\sqrt{2})=C_{2},
$$

puis on peut passer à la limite $m \rightarrow \infty$ en utilisant (3.3).

Pour démontrer le Théorème $(H 2)$, il reste donc à prouver que $R(\beta)$ existe.

Dans ce but, étudions la fonction

$$
F_{j}(\eta)=W_{2^{j}-1}^{j+1}\left(2^{j+1} \eta\right)=M_{0}(\eta) M_{1}(2 \eta) \cdots M_{1}\left(2^{j} \eta\right) .
$$

Cette fonction est paire, $2 \pi$-périodique, positive, et le support de sa restriction à $[0, \pi]$ est inclus dans l'intervalle $[\pi / 6,2 \pi / 3]$.

On appelle $d_{1}$ l'homothétie de rapport négatif qui transforme l'intervalle $[0,1]$ en l'intervalle $[\pi / 6, \pi / 3]$, et $d_{2}$ l'homothétie de rapport positif qui transforme $[0,1]$ en $[\pi / 3,2 \pi / 3]$.

Soit $\mu$ la fonction périodique de période 4 telle que pour tout $x$ dans l'intervalle $[0,4]$ on ait $\mu(x)=M_{0}(\pi(1+x) / 3)$. D'après l'hypothèse $\left(H_{2}\right), \mu$ est continue sur $\mathbb{R}$, s'annule sur $[1,3]$, est symétrique par rapport à 2 , et vérifie l'inégalité

$$
0 \leq \mu(x) \leq \min \{1,3 \beta(1-x)\}, \quad \text { pour tout } x \in[0,1] .
$$


On pose $f_{j}(x)=\mu(x) \cdots \mu\left(4^{j} x\right)$ pour $x$ dans $[0,1]$. Par récurrence sur $j$, on déduit aisément les égalités suivantes de l'hypothèse $\left(H_{2}\right)$ :

$$
F_{2 j} \circ d_{2}=F_{(2 j+1)} \circ d_{2}=F_{(2 j+1)} \circ d_{1}=F_{(2 j+2)} \circ d_{1}=f_{j} .
$$

$E(r)$ désignant la partie réelle d'un réel $r$, on note, pour $h>1$ et $x \in\left[0, h / 4^{j+1}\right]$,

$$
\sigma_{j, h}(x)=\sum_{k=0}^{E\left(4^{j+1} / h\right)-1} f_{j}\left(x+\frac{h k}{4^{j+1}}\right) .
$$

D'après (4.10), on a

$$
\left\{\begin{array}{l}
\sup _{[0, \pi]} \Sigma_{2 j} \leq 2 \sup _{\left[0,6 / 4^{j}\right]} \sigma_{j-1,6}+2 \sup _{\left[0,3 / 4^{j}\right]} \sigma_{j, 12} \\
\sup _{[0, \pi]} \Sigma_{2 j+1}=2 \sup _{\left[0,3 / 4^{j}\right]} \sigma_{j, 12}+2 \sup _{\left[0,6 / 4^{j+1}\right]} \sigma_{j, 6}
\end{array}\right.
$$

Pour trouver $R(\beta)$, il faut donc étudier $f_{j}$. Dans ce but, on décompose $x$ en base $4, x=\sum_{q \geq 1} \varepsilon_{q} / 4^{q}, 0 \leq \varepsilon_{q} \leq 3$. Le support de $f_{j}$ est inclus dans l'ensemble de Cantor des $x$ de la forme $\sum_{q \geq 1} 3 \alpha_{q} / 4^{q}, \alpha_{q} \in\{0,1\}$.

Nous allons majorer $f_{j}(x)$ pour $x$ de cette forme, par un calcul similaire à [6, p. 102].

Avec la notation $\alpha_{0}=1$, on considère la suite $0 \leq q_{1}<q_{2}<\cdots$ des indices tels que $\alpha_{q} \neq \alpha_{q+1}$.

Pour $j$ fixé, on appelle $I$ l'indice maximal tel que $q_{I} \leq j$, et on pose $l_{1}=q_{2}-q_{1}, \ldots, l_{I}=(j+1)-q_{I}$. Prenons maintenant un entier $i$ entre 0 et $I$.

Si $\alpha_{q_{i}}=1$, alors $\alpha_{q}=0$ pour tout $q_{i}<q \leq q_{i+1}$, par conséquent $4^{q_{i}} x \in\left[3,3+4^{-l_{i}}\right] \bmod (4)$.

Si $\alpha_{q_{i}}=0$, alors $\alpha_{q}=1$ pour tout $q_{i}<q \leq q_{i+1}$, par conséquent $4^{q_{i}} x \in\left[3,3+4^{-l_{i}}\right] \bmod (4)$.

Dans les deux cas, on trouve $\mu\left(4^{q_{i}} x\right) \leq 3 \beta 4^{-l_{i}}$, d'où

$$
f_{j}(x) \leq(3 \beta)^{I} 4^{-\left(l_{1}+\cdots+l_{I}\right)}=(3 \beta)^{I} 4^{q_{1}-(j+1)} .
$$

Il en résulte l'existence d'une constante $\rho(\beta)>1$ telle que pour tout $h>1$, on ait

$$
\sup _{0 \leq x \leq h k / 4^{j+1}} \sigma_{j, h}(x) \leq \rho .
$$


En effet, tout intervalle de type $\left[a / 4^{j+1},(a+1) / 4^{j+1}\right]$ contient au plus un point du type $x+h k / 4^{j+1}$, d'où

$$
\begin{aligned}
\sigma_{j, h}(x) & \leq \sum_{a=0}^{4^{j+1}-1} \sup _{\left[a / 4^{j+1},(a+1) / 4^{j+1}\right]} f_{j} \\
& \leq 1+\sum_{q_{1}=0}^{j} 4^{q_{1}-j-1} \sum_{I=1}^{\left(j+1-q_{1}\right)} C_{j-q_{1}}^{I-1}(3 \beta)^{I} \\
& =1+\sum_{q=0}^{j} 4^{q-j-1} 3 \beta(1+3 \beta)^{j-q} \\
& =1+3 \beta \sum_{q=0}^{j}\left(\frac{1+3 \beta}{4}\right)^{q} \\
& \leq \frac{1+3 \beta}{1-\beta} .
\end{aligned}
$$

Maintenant, en combinant (4.13) aux formules (4.11), on trouve $R(\beta)$ vérifiant (4.8), ce qui démontre le Théorème $(H 2)$.

Pour démontrer les Théorèmes $\left(H_{p}\right), p \geq 3$, on procède de façon similaire.

Sous l'hypothèse $\left(H_{p}\right)$, l'inégalité stricte (4.6) n'est possible que si $f$ a une intégrale non nulle sur $\left[\pi-2 \delta_{p}, \pi+2 \delta_{p}\right]$.

Donc, si $f(\xi)=M_{\varepsilon_{j}}(\xi) M_{\varepsilon_{j-1}}(2 \xi) \cdots M_{\varepsilon_{1}}\left(2^{j-1} \xi\right) g\left(2^{j} \xi\right)$ avec $g$ positive, $2 \pi$-périodique et paire, $f$ ne peut vérifier (4.6) que si la suite $\varepsilon_{1} \cdots \varepsilon_{j}$ est constituée d'un premier bloc qui peut être ou bien

$$
(1 \underbrace{0 \ldots 0}_{(p-2) \text { fois } 0} 1),
$$

ou bien

$$
(\underbrace{0 \ldots 0}_{k \text { fois } 0} 1)
$$

avec $0 \leq k \leq p-1$, suivi d'un certain nombre de blocs de la forme

$$
(1 \underbrace{0 \ldots 0}_{(p-2) \text { fois } 0} 1) .
$$


Par conséquent, si l'on trouve un majorant $R(\beta, p)$ aux sommes de la forme

$$
\Sigma_{j}^{p}(\xi)=\sum_{k=0}^{2^{j p+1}-1} W_{n(j, p)}^{j p+1}(\xi+2 k \pi),
$$

où $\xi \in[0, \pi]$ et

$$
n(j, p)=\sum_{q=0}^{j-1} 2^{q p}\left(1+2^{p-1}\right),
$$

alors $\left\|W_{n}^{m}\right\|_{L^{1}\left(-2^{m} \pi, 2^{m} \pi\right)}$ se laisse majorer par $C_{p}(R(\beta, p))^{N_{p}(n)+1}$, avec

$$
C_{p}=2 \pi+4 \delta_{p}(\sqrt{2}-1) \geq 2 \int_{-\pi}^{\pi} M_{0}(\xi) d \xi
$$

Etudions donc $F_{j}^{p}(\eta)=W_{n(j, p)}^{j p+1}\left(2^{j p+1} \eta\right)$, pour $j \geq 1$.

Le support de sa restriction à $[0, \pi]$ est inclus dans l'intervalle

$$
I=\left[\frac{\pi}{2}-\left(1+2^{-(j-1) p}\right) \delta_{p}, \frac{\pi}{2}+\delta_{p}\right]=I_{1} \cup I_{2},
$$

avec

$$
I_{1}=\left[\frac{\pi}{2}-\left(1+2^{-(j-1) p}\right) \delta_{p}, \frac{\pi}{2}-\delta_{p}\right]
$$

et

$$
I_{2}=\left[\frac{\pi}{2}-\delta_{p}, \frac{\pi}{2}+\delta_{p}\right] .
$$

Soit $D$ l'homothétie de rapport positif qui envoie l'intervalle $[0,1]$ sur l'intervalle $I_{2}$. Pour $x \in[0,1]$, on pose $f_{j}^{p}(x)=F_{j}^{p} \circ D(x)$. Etant donnés $h>1$ et $x \in\left[0, h / 2^{(j+1) p}\right]$, on note

$$
\sigma_{j, h}^{p}(x)=\sum_{k=0}^{E\left(2^{p(j+1)} / h\right)-1} f_{j}^{p}\left(x+\frac{h k}{2^{p(j+1)}}\right) .
$$

L'intervalle $I_{1}$ peut contenir au plus un réel du type $2^{-(j p+1)}(\xi+2 k \pi)$, pour $\xi$ fixé et $k$ variable. Par conséquent, on a l'inégalité

$$
\sup _{\xi \in[0, \pi]} \Sigma_{j}^{p}(\xi) \leq 1+2 \sup _{x \in\left[0, h(p) / 2^{(j+1) p}\right]} \sigma_{j, h(p)}^{p}(x)
$$

avec $h(p)=\pi / \delta_{p}=2\left(2^{p}-1\right)>1$. 
D'après l'hypothèse $\left(H_{p}\right)$, on a la formule

$$
f_{j}^{p}(x)=\nu(x) \nu\left(2^{p} x\right) \cdots \nu\left(2^{j p} x\right)
$$

pour tout $x \in[0,1], \nu$ étant une fonction positive, continue, paire et de période $2^{p}$, s'annulant sur $\left[1,2^{p}-1\right]$, et vérifiant, pour tout $x$ dans $[0,1]$, l'inégalité

$$
\nu(x) \leq \min \left\{1, \beta\left(2^{p}-1\right)(1-x)\right\} .
$$

On voit donc que la fonction $f_{j}^{p}$ a son support inclus dans l'ensemble de Cantor des $x$ de la forme $\sum_{q \geq 1}\left(2^{p}-1\right) \alpha_{q} / 2^{q p}$, avec $\alpha_{q} \in\{0,1\}$, et on trouve une généralisation de (4.12):

$$
f_{j}^{p}(x) \leq\left[\left(2^{p}-1\right) \beta\right]^{I} 2^{p\left(q_{1}-j-1\right)} .
$$

Puis on obtient, pour $h>1$,

$$
\begin{aligned}
\sigma_{j, h}^{p} & \leq 1+\left(2^{p}-1\right) \beta \sum_{q=0}^{j}\left(\frac{1+\left(2^{p}-1\right) \beta}{2^{p}}\right)^{q} \\
& \leq 1+\frac{2^{p} \beta}{1-\beta} .
\end{aligned}
$$

En conclusion, d'après (4.14) et (4.18), on peut prendre

$$
R(\beta, p)=3+\frac{2^{p+1} \beta}{1-\beta},
$$

et le Théorème $\left(H_{p}\right)$ est démontré.

Remerciements. L'auteur remercie Yves Meyer et Ronald Coifman pour leurs conseils et leurs encouragements.

\section{References.}

[1] Cohen, A., Ondelettes, analyses multirésolutions et traitement numérique du signal. Masson, 1992.

[2] Coifman, R. R. et Meyer, Y., Orthonormal wave packet bases, technical report. Yale University, New Haven, 1990. 
[3] Coifman, R. R., Meyer, Y., Quake, S. et Wickerhauser, V. M., Signal processing and compression with wavelet packets. Preprint, Numerical Algorithms Research Group, Department of Mathematics, Yale University, New Haven, 1990.

[4] Coifman, R. R., Meyer Y. et Wickerhauser V. M., Size properties of wavelet packets. Wavelets, ed. by Beylkin, etc. Jones and Bartlett, 1991.

[5] Fang, X. et Séré, E., Adapted multiple folding local trigonometric transforms and wavelet packets. Appl. and Computational Harmonic Analysis 1 (1994), 169-179.

[6] Meyer, Y., Ondelettes et opérateurs I. Hermann 1990.

[7] Meyer, Y., Ondelettes et applications. Armand Colin, 1992.

Recibido: 25 de septiembre de 1.993

Revisado: 31 de marzo de 1.994

Eric Séré

CEREMADE

Université Paris-Dauphine Place de Lattre de Tassigny 75775 Paris Cedex 16, FRANCE sere@paris9.dauphine.fr 